Daniel Weidner · Sigrid Weigel (Hrsg.)

Benjamin-Studien 1 

Daniel Weidner · Sigrid Weigel (Hrsg.)

Benjamin-Studien 1 
Bibliografische Information der Deutschen Nationalbibliothek

Die Deutsche Nationalbibliothek verzeichnet diese Publikation in der Deutschen Nationalbibliografie; detaillierte bibliografische Daten sind im Internet über http://dnb.d-nb.de abrufbar.

Alle Rechte, auch die des auszugsweisen Nachdrucks, der fotomechanischen Wiedergabe und der Übersetzung, vorbehalten. Dies betrifft auch die Vervielfältigung und Übertragung einzelner Textabschnitte, Zeichnungen oder Bilder durch alle Verfahren wie Speicherung und Übertragung auf Papier, Transparente, Filme, Bänder, Platten und andere Medien, soweit es nicht $\$ \$ 53$ und 54

URG ausdrücklich gestatten.

(C) 2008 Wilhelm Fink Verlag, München

(Wilhelm Fink GmbH \& Co. Verlags-KG, Jühenplatz 1, D-33098 Paderborn)

Internet: www.fink.de

Layout: Marietta Damm, Zentrum für Literatur- und Kulturforschung Berlin Einbandgestaltung: Evelyn Ziegler, München

Printed in Germany

Herstellung: Ferdinand Schöningh GmbH \& Co KG, Paderborn

ISBN 978-3-7705-4637-4 


\title{
Aura di San Pellegrino: Anmerkungen zu Benjamin-Archiv Ms 931 ${ }^{1}$
}

\begin{abstract}
Man darf hier den Menschen wohl bewundern als ein gewaltiges Baugenie, dem auf beweglichen Fundamenten und gleichsam auf fliessendem Wasser das Aufthürmen eines unendlich complicirten Begriffsdomes gelingt[.]
\end{abstract}

Friedrich Nietzsche

Meine Überlegungen auf diesen Seiten drehen sich um ein von Walter Benjamin handbeschriebenes Blatt Papier, an dem sich die performative Dimension von Benjamins Poetik paradigmatisch illustrieren lässt. Das Manuskriptblatt (Benjamin-Archiv Ms 931, vgl. Abbildung) wurde von Gershom Scholem auf ca. 1935 datiert und gehört thematisch in den Umkreis des etwa zwei Jahre vorher verfassten sprachtheoretischen Essays »Lehre vom Ähnlichen«. Dort heißt es, dass die »Einsicht in die Bereiche des ^Ähnlichen` [...] weniger im Aufweis angetroffener Ähnlichkeiten [zu gewinnen sei] als durch die Wiedergabe von Prozessen, die solche Ähnlichkeiten erzeugen« (GS II, 204). In Benjamins Beschriftung von Ms 931 wird dies in die Tat umgesetzt: wir können uns den Schreibprozess, dem sich der handschriftliche Text auf jenem Blatt verdankt, als Selbsterforschung eines solchen Prozesses denken. In dem vorliegenden Versuch scheint jedoch, neben der Erzeugung von Ähnlichkeiten, die »Überwindung des Mythos» (Ms 931) als zweiter Pol am Horizont auf. Zusammen stecken jene beiden Pole ein dialektisches Spannungsfeld ab, in welchem sich sowohl Benjamins Schreibprozess als auch der meinige bewegen, und dessen Gesetzlichkeit das eigentliche Objekt dieser Überlegungen ausmacht.

1 Dieser Essay ist die überarbeitete und erweiterte Version des unter dem gleichen Titel am 23. Oktober 2006 im Rahmen des Berliner Benjamin-Kongresses (Workshop „Edition und Archiv«) gehaltenen Vortrages. Er ist Teil eines in Arbeit befindlichen Buchprojektes zu Benjamins Poetik. Die Forschungsarbeit für diesen Beitrag wurde durch ein Reisestipendium der Arts and Humanities Initiative (AHI) von der University of Iowa für den Sommer 2005 ermöglicht. Die Schreibarbeit wurde während eines Forschungssemesters von der University of Iowa am Obermann Center for Advanced Studies begonnen. Mein Dank geht auch an die Mitarbeiter des Benjamin-Archivs in Berlin, und ganz besonders an Frau Ursula Marx, für ihre immer freundliche Hilfe. Ich möchte auch Joshua Gold und Sandro Zanetti herzlich für ihre kritischen und produktiven Reaktionen auf diesen Essay danken. Ich hoffe, zumindest auf einige ihrer Fragen geantwortet zu haben. 


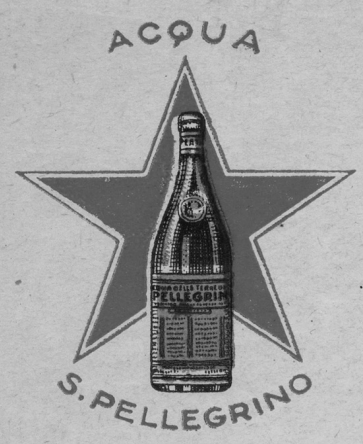

\section{Benjamin-Archiv \\ Ne 931}

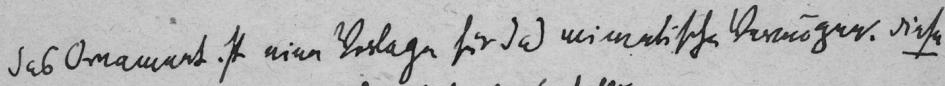

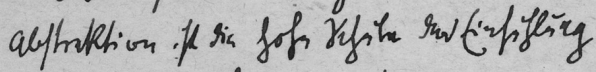

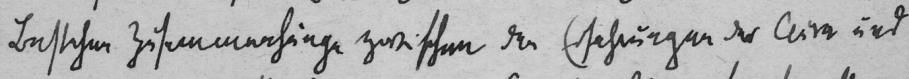

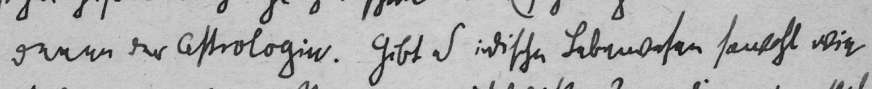

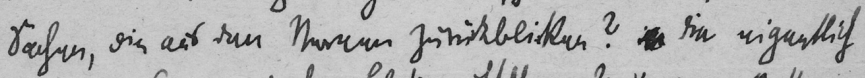

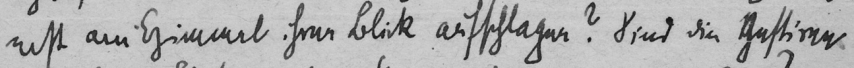

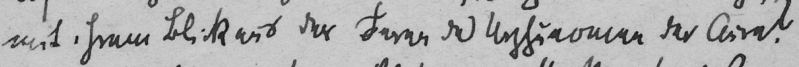

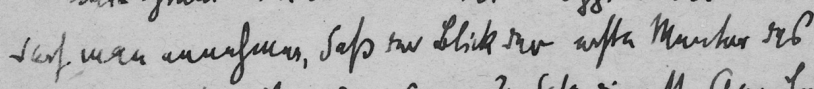

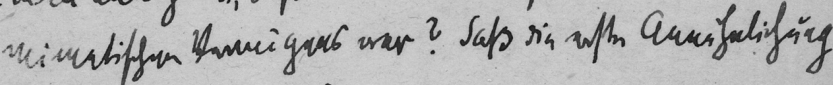
hif im Lhik sollyift?

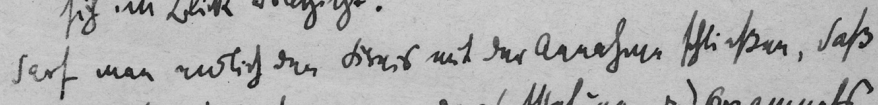

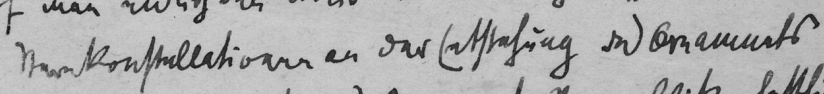

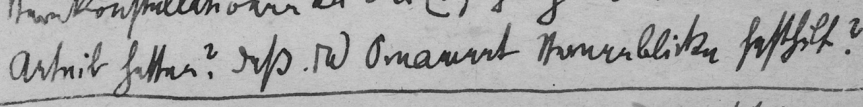

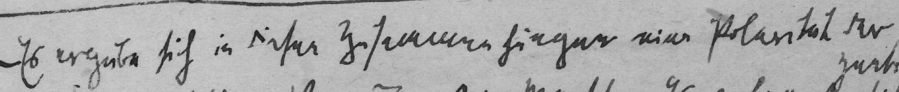

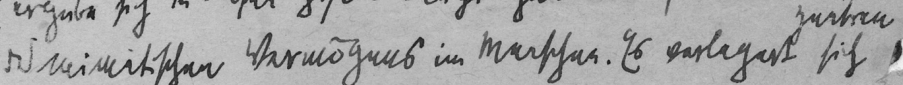

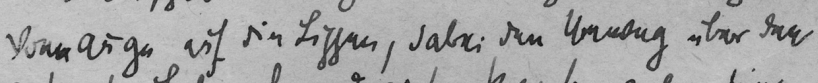

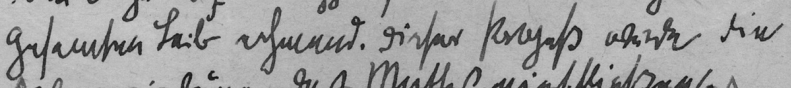

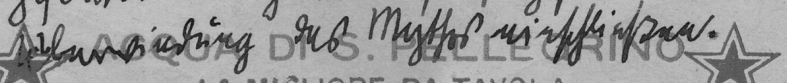
LA MIE:IORE DA TAVOLA 


\section{AURA DI SAN PELLEGRINO}

\section{Lesen und Schreiben}

Benjamin gehörte zu den Papierarbeitern. Die Arbeit am Manuskript war ihm körperlich erlebbares Denken. Der physische Akt des Schreibens bereitete ihm Lust, wie Theodor W. Adorno überliefert hat. Jeden Transfer in ein anderes Medium empfand er als gravierenden Eingriff. [...] Ein Papierarbeiter muss seine Materialien sorgsam auswählen. „Meide beliebiges Handwerkszeug«, fordert er [...] und: »Pedantisches Beharren bei gewissen Papieren, Federn, Tinten ist von Nutzen. $\ll^{2}$

Bei solcher Papierarbeit geht es um mehr als ein bloßes Notieren von Formulierungen und Sätzen, welche beliebig und medienunabhängig in den Druck überführt werden könnten. Es geht um mehr als den Text, um mehr als die Schrift, es geht um das sich an der lesbaren »Fläche« ${ }^{3}$ erfahrende Schreiben selbst. Aber so wichtig der Prozess des Schreibens für Benjamin war, so sehr entzieht sich letztlich doch sein körperliches Erleben des Schreibens unserer Beobachtung: "Was wir beobachten, ist nicht, nie, das Schreiben, sondern sind vielmehr dessen materielle Spuren, die Schrift. [...] Die Geste aber bleibt uneinholbar. Diese fundamentale Verspätung bildet, als konstitutive Nachträglichkeit, den blinden Fleck aller Reflexionen über das Schreiben. ${ }^{4}$ Doch auch das schreibende Subjekt selbst ist sich nicht ungebrochen präsent: es "wird sich im Spiegel der Schrift nicht als dasselbe schreibende, sondern, im Moment der Wahrnehmung, als immer schon anderes wi(e) derbegegnen. " Auch am Ursprung finden wir demnach also keine Urszene auktorialer Authentizität, sondern nur ein diskontinuierliches Alternieren von Lesen und Schreiben, von Schrift und Deutung. Stephan Kammer unterscheidet zwei gängige Strategien, mit jenem blinden Fleck umzugehen: entweder die "radikale [...] Ausblendung des Komplexes Schreiben-Schrift zugunsten des Texts«, oder umgekehrt die "fetischisierende Überhöhung des die Schreibvorgänge speichernden Manuskripts«. ${ }^{6}$ Was aber, wenn der Unterschied

2 Erdmut Wizisla: „Verzettelte Schreiberei«, in: Trajekte. Zeitschrift des Zentrums für Literatur- und Kulturforschung Berlin 7 (2006) 13, S. 8-12, hier S. 8.

3 Vgl. hierzu das hochinteressante frühe Ms 538, wo Benjamin u.a. schreibt: "Über die Wahrnehmung in sich. [/]Wahrnehmung ist Lesen [/]Lesbar ist nur in der Fläche erscheinendes. [/.../] 3 Fläche die Konfiguration ist - sehr [/] absoluter Zusammenhang«. Zitiert nach Davide Giuriato: "Wahrnehmen und Lesen. Ungelesenes in Walter Benjamins Notiz ,Über die Wahrnehmung in sich", in: Bilder der Handschrift. Die graphische Dimension der Literatur, hg. v. Davide Giuriato/Stephan Kammer, Frankfurt a.M. - Basel (Stroemfeld Verlag), 2006, S. 183-202. (Ms 538 ist sowohl auf S. 200 wie auch auf dem Buchumschlag reproduziert. Der Band wird von nun an als Bilder der Handschrift zitiert.)

4 Stephan Kammer: "Reflexionen der Hand. Zur Poetologie der Differenz von Schreiben und Schrift«, in: Bilder der Handschrift, S. 131-161, hier S. 135.

Ebd., S. 161.

6 Ebd., S. 135. 
zwischen Lesen und Geschriebenem aus dem Sprung, der sie trennt, überhaupt erst hervorginge? Wenn wir uns auch und genau als Lesende nicht weiter vom Ursprung der Schrift entfernt fänden als das Schreiben selbst? Denn Schreiben und Lesen haben dies gemeinsam: dass sie beide im Geschriebenen nicht zu finden sind. Was, wenn das Schreiben in seinem Verschwinden dem Lesen seinen Platz einräumte und einräumen müsste? Angesichts dieser Möglichkeit versucht der vorliegende Essay, jenen blinden Fleck weder auszublenden, noch ihn zu fetischisieren, sondern sich im Gegenteil häuslich in ihm einzurichten: in der ursprünglichen Nachträglichkeit der Schrift - und in der nachträglichen Ursprünglichkeit dieses Lesens. ${ }^{7}$

\section{Das Papier}

Benjamin bevorzugte pedantisch bestimmte Papiere: "Seine private Haltung näherte zuweilen dem Ritual sich an. [...] In den Briefen reicht es bis ins typographische Bild hinein, ja bis in die Wahl des Papiers, das für ihn eine ungemeine Rolle spielte; noch während der Emigrationszeit beschenkte ihn sein Freund Alfred Cohn, wie längst, mit einer bestimmten Papiersorte.$^{8}$ Dessen ungeachtet schrieb Benjamin jedoch auch häufig, und allem Ritual zum Trotz, auf zufällige Zettel, auf alle möglichen Papiere, die eben gerade da waren. "Eine Fülle kleinformatiger Zettel und Blätter, darunter Rückseiten von empfangenen Briefen, Formularen, Rezeptblöcken sowie Anzeigen- und Werbezetteln stehen dafür «. ${ }^{9}$ Auch das Blatt, um das es uns hier gehen soll, lag ihm wahrscheinlich durch Zufall vor. Ms 931 trägt einen Aufdruck, der mit einer Kombination von Werbegrafik und italienischsprachigem Text für das Mineralwasser »Acqua S. Pellegrino« wirbt. Benjamin war Ende 1934 und Anfang 1935, schon zur Zeit seines Pariser Exils, für vier Monate in San Remo, wo die pensione seiner geschiedenen Frau Dora ihm Asyl bot. ${ }^{10}$ Dass er zu jener Zeit auf einem italienischen Reklameblock schreibt, ist also nicht weiter erstaunlich. Soweit der Zufall. Doch so hochgradig bewusst, wie Benjamin sich eben seinem Schreib-

$\mathrm{Zu}$ diesem Thema und vielen anderen in diesen Seiten angesprochenen, vgl. auch: Sabine I. Gölz: The Split Scene of Reading: Nietzsche/Derrida/Kafka/Bachmann, Atlantic Highlands (Humanities Press), 1998.

8 Theodor W. Adorno: „Vorrede«, in: Walter Benjamin: Briefe, hg. v. Gershom Scholem/Theodor W. Adorno, Frankfurt a.M. (Suhrkamp Verlag) 1966, S. 15.

9 Ursula Marx: »In einer so wichtigen Sache als die Verwahrung meiner Papiere،. Das Walter Benjamin Archiv«, in: Trajekte. Zeitschrift des Zentrums für Literatur- und Kulturforschung Berlin 7 (2006) 13, S. 12-14, hier S. 12.

10 Vgl. Gershom Scholem: Walter Benjamin. The Story of a Friendship, Philadelphia (Jewish Publication Society of America) 1981, S. 198-199. 
zeug gegenüber befindet, ist der Gebrauch, welchen er dann von so einem Blatt macht, schon überhaupt nicht mehr zufällig. ${ }^{11}$

Stellen wir uns also einmal den Augenblick vor, in dem Walter Benjamin, dem Papierarbeiter, für den die Begegnung seines Füllers mit einem neuen Schreibpapier eine gewichtige Sache war, dieses Blatt (vielleicht sogar ein ganz neuer Reklameblock im vollen Zauber seiner frischen ersten Seite? vielleicht auf einem Cafétisch in San Remo? womöglich sogar neben einem Glas Mineralwasser?) vorgelegen haben mag. Obwohl noch unberührt von seiner Hand, ist das Blatt, auf das sein Blick da fällt, jedoch nicht leer: auf ihm prangt eine gedruckte Sternenkonstellation. Dass der Text, der dann, in dieser Sternstunde, auf dem Papier entsteht, von Sternen und Astrologie handelt, sowie von Ornamenten, die Blicke festhalten, kann da schon kein Zufall mehr sein. Benjamins Schreiben auf dem Manuskriptblatt Ms 931 ist - diesen Gedanken möchte ich hier durchspielen - mimetische, sich selbst erforschende Beschriftung. Die Handschrift hat ihren Ursprung in einer spielerischen Lektüre des Papiers, auf dem sie steht.

\section{Konstellation}

Sehen wir uns die Konstellation auf dem Blatt genauer an. In der linken oberen Ecke ist ein großer Stern, ${ }^{12}$ der mit dem Wort "Acqua über-, und mit den Worten "S. Pellegrino"

11 Benjamin benützt das hier diskutierte S. Pellegrino Papier noch mehrfach sowie auch zwei weitere Papiere derselben Firma, die ein anderes Design tragen. Eine erste kursorische Sichtung aller in Berlin aufbewahrten, auf solchen Papieren geschriebenen Manuskripte, bestätigt (wenn auch nicht in allen Fällen, so doch) tendenziell die Hypothese, dass das S. Pellegrino Papier im Anschluss an die Beschriftungs- und Lektüreerfahrung von Ms 931 eine besondere Bedeutung für Benjamin hatte, und dass dies sich daran nachweisen lassen sollte, dass er in der Nachfolge jenes Paper gezielt hervorholte, wenn er über bestimmte, mit dem jeweiligen Papier assoziierte Themen schreiben wollte. Die Intensität der schreibenden Auseinandersetzung wie wir sie in Ms 931 beobachten bleibt zwar die Ausnahme. Korrelationen zwischen den Papieren und dem Gebrauch, welchen Benjamin von ihnen macht, scheinen jedoch in der Tat zu bestehen. Eine detaillierte Untersuchung jener anderen Manuskripte wird an anderer Stelle geleistet werden.

12 Der fünfzackige Stern, das Pentagramm, ist ein Symbol mit einer langen Geschichte, auf die ich hier nur kurz hinweise. Es geht 6000 Jahre bis zur babylonischen Astronomie zurück. In der Antike war es mit dem Planeten und der Göttin Venus, mit Gesundheit und Fruchtbarkeit assoziiert. (Wegen der relativen Bahnen von Erde und Venus ergibt die Beschreibung der Bewegungen des letzteren Planeten von der Erde aus gesehen über acht Erdenjahre hinweg ein Pentagramm.) Seit Pythagoras soll es auch den Menschen symbolisieren. Es war, zwischen 300-150 v. Chr., Teil des Siegels der Stadt Jerusalem. Im Mittelalter war das Pentagramm ein magisches Symbol, das Schutz vor bösen Geistern gewährte (vgl. die Szene »Studierzimmer« in Goethes Faust). Die umgekehrte Version, bei der eine Spitze nach unten zeigt, ist das Symbol für den Teufel. Er ist das Symbol des Islam, und fünfzackige Sterne finden sich auf vielen nationalen Flaggen (Marokko, US, EU, u.a.m.), sowie im militärischen Kontext auf den Offiziersuniformen und Kampfflugzeugen verschiedener Länder. (Vgl. Carl G. Liungman: Dictionary of Symbols, Santa Barbara - Denver - Oxford (ABC-Clio), 1991; S. 298-300). Assoziiert mit Fortschritt und dem neuen sozialistischen Menschen, leuchtet der rote Stern seit 1935 vom Spasskaja-Turm des Moskauer Kreml. Der Stern ist assoziiert mit Italien, und bildet auch heute Teil des Emblems der Italienischen Republik. 
unterschrieben ist. Der rote fünfzackige Stern ziert die Mineralwasserflaschen der San Pellegrino Quelle bei Bergamo in Norditalien seit der Gründung der Gesellschaft im Jahre 1899. ${ }^{13}$ Um 1935 wird der Stern für Benjamin aber auch Assoziationen mit dem roten Sowjetstern wachgerufen haben, welcher gerade um jene Zeit auf dem Spasskaja-Turm des Moskauer Kreml installiert wurde. Dies wiederum geht dann mit der Tatsache, dass es sich bei dem Aufdruck auf dem Papier um eine Reklame, also um ein kapitalistisches Phänomen par excellence handelt, eine spannungsvolle Mischung ein. ${ }^{14}$

Die gedruckte Sternenkonstellation besteht aus zwei sehr verschiedenen Komponenten: Links oben haben wir den großen Stern mit der grünen Mineralwasserflasche. Auf deren gedrucktem Etikett ist noch ein winziger zweiter Stern zu sehen, so dass die Flasche gewissermaßen von zwei Sternen in die Mitte genommen wird: ein großer shinter und ein kleiner `vor` der Flasche. Das Bild wird von dem erwähnten unvollständigen Schriftkreis von oben und unten eingeklammert.

Ganz unten auf der Seite treten dagegen zwei gleich große Sterne nach links und rechts auseinander und nehmen die zwei Textzeilen - das größere grüne »Acqua di S. Pellegrino« und das kleinere rote "la migliore da tavola« - zwischen sich.

Die beiden Figuren schaffen völlig verschiedene symbolische Raumzeiten, auf deren Gravitationsfelder der Schreibprozess sensibel reagiert. Die obere Figur entsteht durch vertikale Überlagerung, die untere durch horizontale Polarisierung der Sterne. Die Überlagerungsfigur von kleinem Stern, Flasche, und großem Stern kehrt die Relationen von Nähe und Ferne um. Denn perspektivisch müsste das, was uns näher ist, größer erscheinen. Hier aber ist es umgekehrt: der winzige Funken von einem Stern auf dem Flaschenetikett ist uns am `nächsten`, der größte am ıfernsten`. Was wir vor uns haben ist eine Figur für Aura, die ja bekanntlich durch die Umkehrung von nah und fern, als »Erscheinung einer Ferne, so nah sie sein mag“ (GS II, 378) definiert ist. Auch umgibt der große Stern die Flasche wie eine strahlende sternförmige Aura, und die schreibende Hand respektiert ebenfalls die Unnahbarkeit jener Figur: die Zeilen halten Distanz zu dem Bild. Und schließlich kann die Figur auch noch als Darstellung einer explosiven Entwicklung des kleinen Funken hier in der Tiefe der Zeit (großer Stern) gelesen werden. Die zwei kleineren Sterne am unteren Blattrand haben dagegen keine solche Tiefenwirkung. Sie

13 Mein herzlicher Dank geht an Herrn Otto Petracco bei S. Pellegrino in Milano für seine freundliche Hilfe und den Überblick über die Geschichte der S. Pellegrino Quelle und Mineralwassergesellschaft während unseres Telefonats am 11. Januar 2006.

14 Dieses Thema wird auf Ms 931 nicht entfaltet. Dass es dennoch latent vorhanden ist, zeigt sich daran, dass zumindest die Frage der Klassenverhältnisse auf anderen Manuskripten, die auf dem gleichen Papier geschrieben sind, eine prominente Rolle spielt (vgl. besonders Ms 224). 
etablieren einen euklidischen Raum, flach wie jene besten Tische der Welt, auf welche die globalen Aspirationen der Mineralwassergesellschaft zielen, ohne auratische Verwerfungen, ohne dynamische Kraftfelder oder massive Krümmungen, aber auch ohne jene spezifische Mischung von Anziehungskraft und Unnahbarkeit, welche Aura kennzeichnet. Wie um dies zu bestätigen, halten die handgeschriebenen Zeilen auch keine Distanz, sondern eine ganze Schriftzeile legt sich respektlos über den linken Stern und die gedruckte grüne Schriftzeile. Und doch unterwirft sich das Handgeschriebene letztlich dem zweiten (roten) Rahmen, den Sterne und Druckschrift ihm am Seitenende vorgeben.

Auch die gedruckte Schrift verhält sich unterschiedlich. In der oberen Figur deutet die Druckschrift einen fehlschlagenden Versuch an, einen Kreis um das Sternbild zu ziehen, als versuche sie, den in alle fünf Himmelsrichtungen auseinanderstrebenden Stern einzudämmen. Der Behälter Schrift, welcher das Ensemble von Sternen und Flasche noch einmal zu umfangen sucht, bricht auseinander in die Worte "acqua« (,Wasser`) und »s. pellegrino» (’hl. Wanderer`). In der unteren Figur dagegen versuchen sich die gedruckten Schriftzeilen nicht mehr als Behälter, sondern treten im Gegenteil zwischen die Sterne, welche jetzt umgekehrt den Rahmen abgeben, und treiben sie auseinander. Die Schrift wechselt also vom fehlschlagenden Versuch, als Behälter zu fungieren und die Sterne zu ergreifen, zu dessen Gegenteil: dem Impuls der Trennung und des Aufschubs. ${ }^{15}$

\section{Der handgeschriebene Text}

Was schreibt Benjamin nun auf dieses Papier? Schon der erste Blick zeigt, dass das Blatt keine Kladde ist. Es gibt fast keine Korrekturen. Man sieht der Schrift die Zuversicht in die Einsicht des Momentes an. In ihr spiegelt sich Selbstvertrauen. Jede Zeile findet ihren Platz.

Der Text ist in fünf Absätze aufgeteilt, deren Anfangszeilen nach Benjamins Gewohnheit jeweils links etwas überstehen. Der erste und letzte beanspruchen rhetorisch einleitende beziehungsweise abschließende Funktion. Sie umrahmen die drei mittleren Absätze, welche ausschließlich aus Fragesätzen bestehen, und deren mittlerer auch der zentrale Absatz des ganzen kleinen Textes ist. Eine horizontale Linie markiert einen Bruch im Übergang zum letzten Absatz:

15 Auch die Farben verdienen Beachtung: die Farbe Grün ist nur zweimal zu finden. Sie ist beide Male mit Wasser assoziiert (die Flasche, bzw. die Worte ACQUA DI S. PELlEGRINO), beide in Positionen, wo sie von Sternen eingerahmt sind. Alle Rahmen, Schrift oder Sterne, sind rot. 
Das Ornament ist eine Vorlage für das mimetische Vermögen. Diese Abstraktion ist die hohe Schule der Einfühlung

Bestehen Zusammenhänge zwischen den Erfahrungen der Aura und denen der Astrologie. Gibt es irdische Lebewesen sowohl wie Sachen, die aus den Sternen zurückblicken? die eigentlich erst am Himmel ihren Blickaufschlagen? Sind die Gestirne mit ihrem Blick aus der Ferne das Urphänomen der Aura?

Darf man annehmen, daß der Blick der erste Mentor des mimetischen Vermögens war? daß die erste Anähnlichung sich im Blick vollzieht?

Darf man endlich den Kreis mit der Annahme schließen, dass Sternkonstellationen an der Entstehung des Ornaments Anteil hatten? daß das Ornament Sternenblicke festhält?

Es ergäbe sich in diesen Zusammenhängen eine Polarität der Zentren mime tischen Vermögens im Menschen. Es verlagert sich vom Auge auf die Lippen, dabei den Umweg über den gesamten Leib nehmend. Dieser Prozess würde die Überwindung des Mythos einschließen. ${ }^{16}$

Der handgeschriebene Text reagiert sensibel auf die Konfiguration, welche ihm als Vorlage dient. Das Blatt muss bewusst als Ganzes gelesen werden, als ein kleines Werk, dem visuelle, graphische und sprachliche Elemente gleichermaßen integral sind: als Spielzeug für die Phantasie.

\section{Erster Absatz}

„Das Ornament ist eine Vorlage für das mimetische Vermögen." (Ms 931) Der Schreibprozess beginnt im Blick auf das Ornament, und mit der Entscheidung, das Blatt zur Vorlage zu nehmen. Dieser An-Satz erklärt das noch unbeschriebene Blatt zum Spielraum für eine kleine Demonstration. Ein bewusst mimetischer Prozess, der sich seiner Vorlage gegenüber konstituiert, setzt sich in Gang. ${ }^{17}$

16 Die hier wiedergegebene Transkription des handgeschriebenen Textes weicht etwas von dem in GS publizierten Text ab: dort haben die Herausgeber den fehlenden Punkt am Ende des ersten Abschnitts kommentarlos ergänzt, sowie das fehlende Fragezeichen im zweiten Abschnitt in eckigen Klammern eingefügt; anstelle von »im Blick« (2. Absatz) steht dort »dem Blick«; und anstelle von "Zentren mimetischen Vermögens" steht dort "Zentren des mimetischen Vermögens« (GS II, 958). Auch die Einrückungen sowie die horizontale Linie sind dort nicht berücksichtigt.

17 In einem von Scholem auf das gleiche Jahr datierte, wohl kurz vor oder nach Ms 931 geschriebenen Notiz schreibt Benjamin zu diesem Thema: "Zum mimetischen Vermögen[.] Das Ornament steht dem Tanz nahe. Es stellt einen Lehrgang zur Erzeugung von Ähnlichkeit dar. (Man müßte Worringers ,Abstraktion und Einfühlung« heranziehen).« (Benjamin-Archiv Ms 927; vgl. GS II, S. 957) 
Sobald der erste Satz auf das Blatt geschriebenen ist, verändert sich jedoch schon das Arrangement: waren da zunächst nur zwei `Schichten « - die Vorlage (das Blatt mit dem Vordruck) und der selbstbewusst lesende und spielende Prozess, der sich an der Vorlage konstituierte - so erscheint nun der handschriftliche Text als dritte Schicht zwischen jenen beiden.

Das erste Wort des zweiten Satzes ist unterstrichen: "Diese Abstraktion ist die hohe Schule der Einfühlung «. Das unterstrichene Wort deutet emphatisch. Aber wohin deutet es? Im vorausgegangenen Satz, so wie er geschrieben steht, wird keine >Abstraktion erwähnt, auf die zurückverwiesen werden könnte. Selbst wenn wir annähmen, das Wort deute auf den vorausgegangenen Satz zurück, müssten wir also das, worum es dort ging, als eine Form von Abstraktion neu lesen. Wir müssten also die Abstraktion, auf die der Satz uns verweist, wiederholend noch einmal neu ins Werk setzen. Deutete der Richtungspfeil des ersten Satzes gewissermaßen "von oben « auf das Papier und das Ornament als Vorlage "hinunter«, so verweist uns der zweite Satz "nach oben « auf das mimetische Vermögen, das sich als "hohe Schule« selbst benennt als die 'Schicht`, die an der Vorlage zu sich kommt, die aber nie im Geschriebenen selbst zu finden ist. Jene emphatische Deutung gelingt also nur, wenn ich meine eigene abstrahierende Deutungshandlung als die gemeinte erkenne. Obwohl diese Abstraktion also nicht geschrieben werden kann, wird die Wiederholung als Double-Take, die ihre Lektüre erfordert, doch im Text markiert: um das Deiktikum diese zu unterstreichen, muss der Schreibprozess rekursiv verdoppelnd über das Geschriebene zurückgehen und die Linie unter das Wort ziehen. Mit dieser Unterstreichung fällt die Nachträglichkeit wiederholend und bedeutend in den Text ein, der Lese- und Schreibprozess spiegelt sich an der neu hinzugekommenen Schicht des Geschriebenen, holt sich aber sofort wieder in und als diese Abstraktion aus der Schrift zurück. Diese Abstraktion besteht auf ihrer konstitutiven Differenz von der lesbaren Fläche, als »hohe Schule« eines indexikalischen Bewusstseins am Text. Das Geschriebene wird ihr zum Spiegel. ${ }^{18}$ Und Spiegel deuten nicht, sie kehren nur die Richtung um, sodass, wer deutend vor ihnen steht, auf sich selbst zurückverwiesen ist. Einfühlung ist nicht mehr Einfühlung in die Vorlage per se, sondern Einfühlung des lesenden Blickes, der sich mimetisch auf jene einlässt, in seine eigene mimetische Aktivität. ${ }^{19}$ Hier sind alle Richtungen verkehrt, Nachträglichkeit von Vorgängigkeit nicht mehr zu unterscheiden.

18 Vgl. GS VII, S. 666: „Der Nachahmende macht eine Sache scheinbar«.

19 Der Satz enthält auch einen offensichtlichen Verweis auf Wilhelm Worringer. Die emphatische Unterstreichung setzt diese Abstraktion als hohe Schule vielleicht auch von (impliziert: jenem) Begriff der Abstraktion bei Worringer ab. Denn in Worringers Buch sind Abstraktion und Einfühlung als "Pol[e] menschlichen Kunstempfindens« zunächst Gegenbegriffe. Bei Benjamin schlagen die Gegensätze ineinander um. Abstrak- 


\section{Zweiter Absatz}

In den nächsten drei Absätzen lässt sich der mimetisch-einfühlende Prozess zunehmend auf die Perspektive des unter der Feder des Schreibenden hervorwuchernden Geschriebenen ein: »Bestehen Zusammenhänge zwischen den Erfahrungen der Aura und denen der Astrologie. Gibt es irdische Lebewesen sowohl wie Sachen, die aus den Sternen zurückblicken? die eigentlich erst am Himmel ihren Blick aufschlagen? Sind Gestirne mit ihrem Blick aus der Ferne das Urphänomen der Aura? (Ms 931; vgl. GS II, 958) Mit dem spiegelnden diese ist die Umkehrbarkeit der Richtungen in den Text gefallen: es ist nicht mehr klar, ob von oben herab oder von unten hinauf geblickt wird, wer oder was da wo die Augen aufschlägt. Sterne, Blicke, irdische Wesen und Sachen, oben und unten, nah und fern, Wahrnehmungen und Wahrgenommenes überlagern sich. Die Blicke scheinen (sich) im Text auf. Die Fragen im zweiten Absatz stellen sich nicht mehr aus der Perspektive des souverän lesenden Blickes, sondern vielmehr aus der des Textes, der sich in vielfach bedeutende "Zusammenhänge« gestellt weiß, aus denen er zwar hervorgeht, ohne jene aber erfassen zu können. Zwischen der Sternkonstellation auf dem Papier und der agilen Performanz dieser Abstraktion wächst der Text unter der Feder hervor, zwischen Nachahmung und Vorlage, selbst schon potentielle Vorlage für erneute Nachahmung, eigentlich aber ein Drittes, mit seiner eigenen, aus seiner Zwischenstellung erwachsenden Sicht der Dinge. Seine Frage nach den Zusammenhängen zwischen Vorlage und mimetischem Prozess, welchen er sich verdankt, ist die Frage nach seiner Bedeutung, nach seinem Ursprung. Er findet aber nur seine eigene (aus jener Zwischenstellung erwachsende) Unentscheidbarkeit am Himmel gespiegelt.

Aber noch etwas ist da geschehen. Was den Blick auf dieses Manuskriptblatt so suggestiv und fesselnd macht ist ja der Gedanke, dass da eine Sternenkonstellation (die ja

\footnotetext{
tion wird als hohe Schule, also als Gipfel der Einfühlung betrieben, stellt aber weiterhin auch deren Gegenbewegung dar. Benjamin erwähnt Worringers Buch in dem schon zitierten Ms 927 (vgl. Anmerkung 17). Dennoch sind jedoch viele Elemente, die an und auf Benjamins Blatt im Spiel sind, auch bei Worringer schon zu finden. (Wilhelm Worringer: Abstraktion und Einfüblung. Ein Beitrag zur Stilpsychologie, München (Piper), 1948 [zuerst 1908], S. 16-17: "Denn der Grundgedanke unseres Versuches ist, zu zeigen, wie diese moderne Ästhetik, die vom Begriffe der Einfühlung ausgeht, für weite Gebiete der Kunstgeschichte nicht anwendbar ist. Sie hat ihren archimedischen Punkt vielmehr nur auf einem Pol menschlichen Kunstempfindens. Zu einem umfassenden ästhetischen System wird sie sich erst dann gestalten, wenn sie sich mit den Linien, die vom entgegengesetzten Pol herkommen, vereinigt hat. Als diesen Gegenpol betrachten wir eine Ästhetik, die anstatt vom Einfühlungsdrange des Menschen auszugehen, vom Abstraktionsdrange des Menschen ausgeht. Wie der Einfühlungsdrang als Voraussetzung des ästhetischen Erlebens seine Befriedigung in der Schönheit des Organischen findet, so findet der Abstraktionsdrang seine Schönheit im lebenverneinenden Anorganischen, im Kristallinischen oder allgemein gesprochen in aller abstrakten Gesetzmäßigkeit und Notwendigkeit."
} 


\section{AURA DI SAN PELLEGRINO}

- wer weiß? - womöglich das Geheimnis meines Schicksals birgt ...), in einer Drehung um $180^{\circ}$ vom Himmel geholt und in die lesbare Fläche ${ }^{20}$ gebannt wird, wo sie uns nun, mit "mimetische[m] Objektcharakter ${ }^{21}$ begabt, vorliegt. Diese buchstäblich revolutionäre Umkehrung der Verhältnisse, diese kosmische Rotation, welche die Welt auf den Kopf stellt, welche das Blatt so schön und suggestiv illustriert, und deren Implikationen für Machtfragen und Lesbarkeit sehr tief gehen, genau jene Umkehrung also wird in jenem zweiten Absatz zunehmend rückgängig gemacht! Der zweite Absatz hat nichts mehr von der Souveränität des ersten. Er ist geschrieben und weiß sich deshalb gelesen, Sterne wie Blicke sind wieder entrückt, und es bedarf erneut der Astrologie, um die "Zusammenhänge«, in deren Aura er steht, zu deuten. (Es ist auch höchst signifikant, dass die drei mittleren Absätze keine Deiktika enthalten!) Die nächsten beiden Absätze wenden sich nun jeweils einer der Instanzen zu, von denen sich jenes Geschriebene eingerahmt weiß: im nächsten und dritten Absatz geht es um Problematik des Blickes, im vierten um die des Ornaments.

\section{Dritter Absatz}

Blicke sind das ausschließliche Thema des nächsten (und zentralen) Absatzes. Keine Spur von Materialität ist hier mehr übrig: „Darf man annehmen, daß der Blick der erste Mentor des mimetischen Vermögens war? daß die erste Anähnlichung sich im Blick vollzieht?» (Ms 931; vgl. GS II, 958) Der Text wendet sich den Blicken zu, im Versuch, dem mimetischen Leseprozess auf die Spur zu kommen, dessen Niederschlag er selbst ist, und den er doch nicht fassen kann. In diesem Zenit der Immaterialität, wo Blick nur noch von Blick lernt, erahnt er den Kern des mimetischen Prozesses. Das Wort »Blick« erscheint zweimal, immer im Singular. Doch dennoch ist da etwas doppelt. Denn der Satz unterscheidet zwei Temporalitäten (»war« im Imperfekt; »vollzieht« im Präsens), sowie zwei Funktionen (im Blick wird das Ähnlichen sowohl gelehrt als auch gelernt). Die Schicht des Blickes wird gespalten, eine hierarchische Differenz zwischen vergangenem und gegenwärtigem, zwischen Mentor- und Schüler-Blick konstruiert. Das mimetische Vermächtnis wird nicht nur im Blick, sondern mit Hilfe einer Hierarchie der Blicke weitergereicht.

Benjamins Neologismus "Anähnlichung" ist ebenfalls bedenkenswert. Da wird das Präfix ıan-^ einem Wort angefügt, in dessen Wurzel selbst bereits die Präposition `an` ent-

Vgl. Anm. 3.

21 Vgl. GS II, S. 206: „Wir müssen nämlich als Erforscher der alten Überlieferungen damit rechnen, daß sinnfällige Gestaltung, mimetischer Objektcharakter bestanden habe, wo wir ihn heute nicht einmal zu ahnen fähig sind. Zum Beispiel in den Konstellationen der Sterne." 
halten ist. Wenn wir dem Duden Glauben schenken, so ist jene Präposition nämlich die Urahne einer ganzen Ansammlung von Worten wie Ahnenschaft, ähnlich, Ähnlichkeit, Ahnung, ahnen, aber auch ahnden, dann stammen all jene Worte von der Präposition ran her, welche eine Berührung durch etwas konnotiert, das uns von außen her ankommt, tangential, ohne vollständig oder deutlich wahrnehmbar zu werden. ${ }^{22}$ Wenn Benjamin

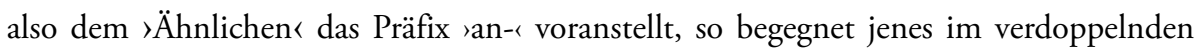
‘an-ähn-` gespiegelt einer älteren Version von sich selbst. Der Blick erkennt, wie Narziss, sein Vor-bild an, er ähnlicht sich an. Oder aber: das, was hier lesend am Werk ist, wird genau in der minimalen Differenz zwischen ran` und ıähn-ヶ seiner separaten Existenz am Text bewusst und sieht plötzlich ferner auf jenen zurück. Denn das Wort `Anähnlichung tut des Guten eine Spur zuviel - es erregt Anstoß. Im »An-« wird die Anstrengung des Ähnlichens, wird ein Widerstand spürbar.

\section{Vierter Absatz}

Mit dem vierten Absatz neigt sich der Text nun von den lesenden Blicken weg, und zum anderen Extrem: zurück zur Vorlage, zum Ornament, nun mit einem angeähnlichten Blick sozusagen schon im Gepäck: "Darf man endlich den Kreis mit der Annahme schließen, dass Sternkonstellationen an der Entstehung des Ornaments Anteil hatten? daß das Ornament Sternenblicke festhält?« (Benjamin-Archiv Ms 931; vgl. GS II, 958) Den Kreis schließen könnte die `Annahme - d.h. die Vermutung oder deren Akzeptanz - dass Sternenkonstellationen "an der Entstehung des Ornaments Anteil hatten«, dass es im Ornament (und im Text) einen tieferen Sinn zu suchen gäbe, der uns zu den Konstellationen an seinem Ursprung zurückführte. Nur dieses wandernde lesende Auge könnte jedoch die

22 Vgl. der Eintrag zum Wort "an« in: Duden. Das Herkunftswörterbuch. Etymologie der deutschen Sprache, 2., völlig neu bearbeitete und erweiterte Auflage von Günther Drosdowski, Mannheim - Leipzig - Wien - Zürich (Duden Verlag) 1989, S. 34: »an: Das gemeingerm. Wort (Präposition und Adverb) mhd. an[e], ahd. an[a], got. ana, engl. on, schwed. å beruht mit verwandten Wörtern in anderen idg. Sprachen auf $i d g$. *an- an etwas hin oder entlang`, vgl. z.B. griech. aná ‘auf, hinan, entlang`, das in zahlreichen aus dem Griech. entlehnten Wörtern als erster Bestandteil steckt. [...] Zu ran«stellen sich im Dt. die Bildungen •ahnen und •ähnlich. Als Adverb ist san ‘ durch 'heran ‘ und 'hinan ‘ ersetzt worden, steckt aber in unfest zusammengewachsenen Verben und Wörtern wie bergan, hintenan, anbei.« Ebenda, S. 25: „ahnen, voraussehen, unmittelbar empfinden, vermuten : Das nur $d t$. Verb ( $m h d$. anen) ist wahrscheinlich von der unter • an behandelten Präposition abgeleitet und bedeutet demnach eigentlich seinen an- oder überkommen`. Es wurde zunächst unpersönlich gebraucht, beachte mhd. ses anet mir (auch: mich)^ ses kommt mich an`, d.h. etwas Unbestimmtes rührt mich von außen her an. Abl.: Ahnung sunbestimmtes Gefühl, Vermutung` (17. Jh.)." (Carol Jacobs weist in ihrem Essay über die "Lehre vom Ähnlichen" auf die Etymologie des Wortes "ähnlich" hin. Vgl. Carol Jacobs: In the Language of Walter Benjamin, Baltimore - London [Johns Hopkins University Press] 1999, S. 91f.) 
Annahme vollziehen. Wird der Kreis aber nicht geschlossen, dann bleibt die Frage offen, dann ist das Geschriebene nicht nur unvollständig, sondern auch instabil: es kann dann keine Notwendigkeit beanspruchen, die Ähnlichkeiten könnten das nächste Mal eine völlig andere, neue Formen annehmen. Das Geschriebene aber, da es nun schon einmal da ist, wünscht sich Notwendigkeit, es wünscht sich Inhalt und Dauer. Es wünscht sich die Annahme einer Wahrheit, es wünscht, dass das Text-Ornament Sehweisen kodiere, denen der Blick immer schon nach-las und weiter nach-lesen soll. Denn dann könnte das, was da, zum Beispiel, aus der zufälligen Begegnung eines Blickes und eines Füllers mit einem Papier hervorging, mit wachsender Notwendigkeit weiterleben und sich reproduzieren.

Und so entsteht im vierten Absatz noch einmal eine hierarchische, temporalisierte Verdoppelung in zwei Schichten: eine frühere Schicht, wo Sternenkonstellationen an der Entstehung des Ornaments »Anteil« haben, und eine, wo Sternenblicke, die früher, jetzt und in Zukunft auf das Blatt gefallen sind, fallen und fallen werden, festgehalten werden. Was wir da anzunehmen gebeten werden ist also noch einmal eine Hierarchie des Vorher über das Nachher, den Gedanken also, dass das Lesen immer schon zu spät komme, und so zur (bloßen) Nachträglichkeit verurteilt sei.

Das Geschriebene ist der Mittler, der - sich mal als Vorlage und mal als Blick, mal spiegelnd und mal transparent gebend, - sich unsichtbar zu machen sucht, der aber dennoch und um so mehr im Interesse seiner eigenen Selbsterhaltung agiert. Er arbeitet daran, Zusammenhänge zu weben, Ornamente in Blicke und Blicke in Ornamente zu überführen, das Lesen, welches spielend immer neue schafft, in die Pflicht bestehender Ähnlichkeiten zu nehmen, und jene zu kodieren, zu multiplizieren, zu reproduzieren. Ein Kreis zwischen Vorlage und Nachahmung soll geschlossen werden, wo vor dem Dazwischengehen des Geschriebenen noch keiner war. Denn erst mit dem Geschriebenen ist der Gedanke aufgekommen, hier etwas festhalten zu wollen. ${ }^{23}$ Der Kreis installiert die Rückkoppelung, welche die Autopoiesis der Schrift und der Sprache treibt, und welche jene in »ein Archiv unsinnlicher Ähnlichkeiten, unsinnlicher Korrespondenzen «(GS II, 208) verwandelt. Die Sprache gibt sich als Behälter, in den angeblich etwas Ursprüngliches, direkt von der Quelle, abgefüllt sei.

Aber nehmen wir einmal an, dieser Blick schlösse die Rückkoppelungsschleife wirklich. Auch diese Entscheidung wäre wieder zweifach lesbar.

23 Vgl. Michel de Certau: The Practice of Everyday Life, Berkeley - Los Angeles (University of California Press), 1984 über die "triumphal conquista of the economy that has, since the beginning of the smodern age $[. .$.$] given itself the name of writing «, S. 131, sowie seine Unterscheidung von Strategie und Taktik$ (ebd., S. 29-42). 
Der Mensch, der die Annahme annimmt, "dass Sternkonstellationen an der Entstehung des Ornaments Anteil hatten « (Ms 931) und nach deren Zusammenhängen sucht, findet sich in einem scheinbar grenzenlosen metaphorischen Wirkungsraum wieder, in dem alles Augen hat, weil überall Lektüren codiert, antizipiert und präfiguriert sind, einen von Allegorien des Lesens gespenstisch entstellten Raum, der schließlich unlesbar wird, weil dank der Rekursivität, die ihn geschaffen hat, alles mit allem korrespondiert, und jede neue Lektüre das Gespinst nur weiter verdichtet:

[NB: Dem Baudelaire-Gedicht scheinen in der gedruckten Version zwei Leerzeilen (statt einer) vorauszugehen!]

La Nature est un temple où de vivants piliers

Laissent parfois sortir de confuses paroles;

L'homme y passe à travers des forêts de symboles

Qui l'observent avec des regards familiers.

Comme de longs échos qui de loin se confondent

Dans une ténébreuse et profonde unité,

Vaste comme la nuit et comme la clarté,

Les parfums, les couleurs et les sons se répondent. [... $]^{24}$

Die Annahme, dass Ornamente und Metaphern Blicke festhalten, erscheint in jener Welt natürlich als das sine qua non einer jeden Lektüre. Ohne die Annahme schlösse sich der Kreis ja nicht, können die Lesenden ja nicht von ihrem Mentor lernen, wäre die Magie unterbrochen, schösse der Zauber nicht an. Ohne Assimilation keine Lesbarkeit. Das sprachliche Universum übt einen »gewaltigen Zwang [...], ähnlich zu werden« (GS II, 210) aus. Doch das Umgekehrte wäre ebenfalls der Fall: gerade weil die Leser angeähnlicht sind, und daher immer schon An-Teil haben an dem, was sie sich zu lesen bemühen, können sie nichts mehr lesen. Die Sprache wird zum Mythos. Als angeähnlichte sind unsere Blicke in den Dienst der autopoetischen Fortschreibung des Bestehenden gestellt. Der Kreis wäre der einer ewigen Wiederkehr des Gleichen.

Um da Lesbarkeit wiederherzustellen, müssen wir einen archimedischen Punkt finden, der sich nicht von jenen »forêts de symboles« assimilieren lässt.

24 Charles Baudelaire: „Correspondances«, in: Les Fleurs du Mal et autres poèmes, Paris (Flammarion) 1964, S. 39-40. Siehe auch Paul de Mans einflussreiche Interpretation des Gedichtes in: "Anthropomorphism and Trope in the Lyric", in: The Rhetoric of Romanticism, New York (Columbia University Press) 1984, S. 239-262. 
Erinnern wir uns noch einmal, dass »Einsicht in die Bereiche des `Ähnlichen « [...] weniger im Aufweis angetroffener Ähnlichkeiten [zu gewinnen ist] als durch die Wiedergabe von Prozessen, die solche Ähnlichkeiten erzeugen« (GS II, 204). Was wir beobachtet haben, sind Prozesse, die vermittels Hierarchisierung und Rekursivität Ähnlichkeiten zu reproduzieren streben. Erst im Blick auf jene Prozesse selbst lässt sich der Zwang zur Anähnlichung nicht nur als solcher erfahren, sondern auch wieder lesen. Dann aber könnte sein, dass alles noch ein weiteres Mal umgekehrt wäre. Dass die Annahme und das Schließen des Kreises gar nicht mehr zum Fortschreiben der Ähnlichkeiten, sondern zu einer Art Kurzschluss führte, weil der lesende Blick nicht nur auf der Suche nach ursprünglichen Ähnlichkeiten wirklich an einen Ursprung zurückkehrte: an den Moment, wo an einem zufällig vorgefundenen Papier, welches da den Anlass gab, diese Abstraktion als hohe Schule der Anähnlichung zu sich kam. Wenn sich dieser lesende Blick nicht mehr als nachträglich verstünde, sondern an der Entstehung des Ornaments auch heute Anteil nimmt, dann wird der Bildcharakter des Textes »durchgesengt" (GS II, 37125). Dann kommt es zu jener `Sprengung`, über die es in Benjamins Texten bekanntlich viel (und viel Ambivalentes) zu lesen gibt. ${ }^{26}$

Wenn dem aber so wäre, wenn das Schließen des Kreises auch die Form einer Rückkehr dieser Abstraktion - nicht als verspätete, sondern als unverzichtbare und immer wieder neu ursprüngliche lesende Instanz--in den Ur-sprung nehmen könnte, dann zielte vielleicht jene Frage danach, ob "man« den Kreis schließen dürfe (der Bitte des Mannes vom Lande um Einlass in Kafkas »Vor dem Gesetz« ähnlich ${ }^{27}$ ) gar nicht auf Annahme, sondern umgekehrt (nach dem Motto »Wer viel fragt kriegt viel’ Antworten!«) darauf ab, das Schließen des Kreises unbefristet hinauszuzögern, es aufzuschieben, also die Unentscheidbarkeit und Unvollständigkeit des Textes, der ohne diese lesende Instanz, die ja nie ein ıman` ist, gar nichts bedeuten kann, nicht so sehr zu überwinden, als sie vielmehr auf sein Banner zu setzen und zur Methode zu machen. Dann würde die Frage um Erlaubnis so allgemein gestellt, genau weil sie dann keine Antwort hat und offen bleiben muss - und so den Kurzschluss verhindern kann.

Aber selbst jenen Aufschub kann das Geschriebene natürlich letztlich nicht garantieren.

25 Die bekannte Passage aus der "Kleine[n] Geschichte der Photographie« lautet: "Aller Kunstfertigkeit des Photographen und aller Planmäßigkeit in der Haltung seines Modells zum Trotz fühlt der Beschauer unwiderstehlich den Zwang, in solchem Bild das winzige Fünkchen Zufall, Hier und Jetzt, zu suchen mit dem die Wirklichkeit den Bildcharakter gleichsam durchgesengt hat, die unscheinbare Stelle zu finden, in welcher, im Sosein jener längstvergangenen Minute das Künftige noch heut und so beredt nistet, daß wir, rückblickend, es entdecken können." (GS II, S. 371)

26 Vgl. hierzu: Sabine I. Gölz: "Geistesgegenwart als Lichtung im Ähnlichen: Bachmanns `Heute` oder Benjamins 'Jetztzeit?? in: Re-Acting to Ingeborg Bachmann, ed. Caitriona Leahy/Bernadette Cronin, Würzburg (Königshausen \& Neumann) 2006, S. 97-109.

27 Vgl. meine Lektüre jener Parabel in: The Split Scene of Reading, S. 133-141. 


\section{SABINE I. GÖLZ}

\section{Strich}

Auf dem Blatt folgt auf jene letzte Frage tatsächlich kein Kreis, sondern eine horizontale Linie: ein energischer, über die gesamte Breite des Blattes gezogener Strich. Was tut die dramatische Horizontale auf dem Blatt? Am nächsten verwandt ist ihr die Unterstreichung des Wortes diese, deren kleiner Unter- Strich sie präfiguriert. Sie trennt nun, ganz konkret, das (auf dem Blatt) >obere Regime (kleiner Stern, Flasche, und großer Stern; auratische, metaphorische, anähnlichende Schrift) vom sunteren` Regime (zwei gleich große Sterne; Schrift als Aufschub, différance und Polarisierung). Mit dem Strich interveniert etwas, kommt etwas dazwischen, das dem magischen Kreis der Metaphern und Ähnlichkeiten nicht assimiliert werden kann. Denn die Linie ist weder Schrift noch Metapher, weder Sprache noch Körper. Sie ist die Spur der Kontiguität von Füller und Papier, purer Ausdruck der Materialität des Schreibens, die hier sprachlos ihrer Differenz von der Sprache Ausdruck verleiht. Mit dieser Linie scheiden sich die Geister nicht nur am, sondern auch vom Geschriebenen. Der Strich ist Tangente, die den Kreis zwar berührt und schließt, jedoch von außen, und ohne sich der Herrschaft der Sprache zu beugen. Der Strich, mit dem das Schreiben das Papier tangiert, bringt uns, in dieser Abstraktion betrachtet, in einer kopernikanischen Revolution von den fernen Sternen zurück ins Hiesige: zur Materialität des Schriftzeichens und zu einem Körper.

\section{Fünfter und letzter Absatz}

Die Ferne der Sterne ist der Nähe eines Leibes gewichen. Letzterer, traditionell Ziel der Assimilations- und Biopolitik, erscheint nun unter dem Strich im Text: ${ }^{28}$

Es ergäbe sich in diesen Zusammenhängen eine Polarität der Zentren mimetischen Vermögens im Menschen. Es verlagert sich vom Auge auf die Lippen, dabei den Umweg über den gesamten Leib nehmend. Dieser Prozess würde die Überwindung des Mythos einschließen. (Benjamin-Archiv MS 931; vgl. GS II, 958)

Es läge nahe, zu erwarten, es handle sich bei dem Leib, der da erscheint, wohl um den des Schreibenden, der gerade jene Gerade gezogen hat und sich dabei an sich selbst erinnerte. Doch der »Leib«, der da geschrieben steht, ist kein schreibender: er hat zwar "Auge« und "Lippen«, doch das Organ, das den Strich zog - die schreibende Hand nämlich -

28 Bekanntlich »kennt das älteste Nachahmen zunächst nur einen einzigen Stoff, in dem es bildet; das ist der Leib des Nachahmenden selber« (GS VII, S. 666; Ms 971). 
fehlt ihm gänzlich. Es ist, als wüsste das Geschriebene nichts mehr von Hand und Füller, als hätte der Text das Schreiben, dem er sich verdankt, ganz und gar vergessen.

Zielte der Abschnitt über dem Strich ostentativ darauf ab, den »Kreis« zu schließen, so entwirft der Text unter dem Strich nun einen "Umweg«, so als solle nun die Distanz, welche sich jener zu überwinden bemühte, nun von diesem wieder hergestellt werden. Der Schreibprozess betreibt Trennung - eine Trennung, die noch einmal (wie dort die Anähnlichung der Blicke) auf das Lesen abzielt. Der Prozess setzt ein mit einem Deiktikum (dem ersten seit »diese Abstraktion« den ganzen Prozess in Gang brachte): „Es ergäbe sich in diesen Zusammenhängen [...]«. Anstatt jedoch, wie im ersten Abschnitt, unterstrichen zu werden, wird die Tatsache, dass dieses Wort in mindestens zwei Richtungen zeigt, und dass es deshalb hier zu einer vertikalen Schichtung kommt, nun benannt als »[...] eine Polarität«. Diese Zusammenhänge sollen nun auseinanderdividiert und in einen Plural überführt werden. Die Herstellung jenes Plurals ist erst mit der Schrift, die auch hier dazwischenkommt, geschafft: erst das Wort "Zentren«(das einzige Wort auf dem ganzen Blatt, das etwas kleiner geschrieben zwischen den Zeilen steht, und deshalb wohl mit leichter Verspätung in einer Sofortkorrektur eingefügt wurde) macht aus der bloßen Polarität einen echten Plural: Zentren. Das »mimetische [...] Vermögen [...]« bleibt dabei jedoch weiter im Singular, und die Frage der Polarität hat nun damit zu tun, wohin es sein Gewicht legt: "Es verlagert sich vom Auge auf die Lippen, dabei den Umweg über den gesamten Leib nehmend« (Ms 931; Hvh. S.I.G.).

Mit jenem auf- und auseinanderschiebenden, den Leib in getrennte "Zentren " pluralisierenden Dazwischenkommen gehorcht die Beschriftung auch im fünften Absatz weiterhin dem Kraftfeld, welches ihr die gedruckte Vorlage vorgab. Am unteren Seitenende ist Polarisierung angesagt: zwei kleinere Sterne, durch eine dazwischenkommende Schrift voneinander getrennt. Im Vordruck ist jene Schrift aber nun selbst so doppelt wie es der Blick (im dritten Abschnitt) und das Ornament (im vierten) waren! Denn über der roten Zeile la migliore da tavola steht, in grün: acqua di s. pellegrino. Aber während das Geschriebene jene anderen beiden hierarchischen Verdoppelungen aktiv betrieb, tut es nun das Gegenteil und streicht eine der beiden Schichten aus: die Handschrift legt sich über die grüne Schriftzeile, so als solle jene ausgestrichen werden, als sollten wir auch die Tatsache vergessen, dass alles, was wir hier gelesen haben, auf dem fließenden Wasser und Wandern dieses Prozesses aufbaut; als sollten wir stattdessen unser Selbstbewusstsein im Glauben an die Wahrheiten der flachen Welt der Tische und der lesbaren Flächen verankern: 


\section{SABINE I. GÖLZ}

Nur durch das Vergessen jener primitiven Metapherwelt, nur durch das Hartund Starr-Werden einer ursprünglich in hitziger Flüssigkeit aus dem Urvermögen menschlicher Phantasie hervorströmenden Bildermasse, nur durch den unbesiegbaren Glauben, diese Sonne, dieses Fenster, dieser Tisch sei eine Wahrheit an sich, kurz nur dadurch, dass der Mensch sich als Subjekt und zwar als künstlerisch schaffendes Subjekt vergisst, lebt er mit einiger Ruhe, Sicherheit, und Consequenz; wenn er einen Augenblick nur aus den Gefängniswänden dieses Glaubens heraus könnte, so wäre es sofort mit seinem `Selbstbewusstsein` vorbei. ${ }^{29}$

Doch auch dies ist nur scheinbar. Auch der Aufschub lässt sich wieder (mindestens) zweifach lesen. Wir können ihn in der Tat so lesen, als solle der Leib des Lesenden da von sich selbst entfernt werden, zum bloßen Umweg vom Auge zu den Lippen, vom Nach-Lesen der Schrift zur Nach-Sprechen der Sprache. Dann wäre der lesende Leib noch einmal bloß ein blindes Bindeglied, das den Zusammenhang von Sprache (Schrift) mit Sprache (Sprechen), herstellt, ohne noch von sich zu wissen. Ein solches bloßes Nach-Lesen würde nur den Kreis schließen und die Sprache mit sich selbst rückkoppeln. Es wäre dem Mythos der Sprache ausgeliefert.

Wir können den Text aber auch als eine Anleitung lesen, als Empfehlung, das Gewicht unseres mimetischen Vermögens vom Auge auf diese Lippen zu verlegen. Schien es eben noch, dass das Geschriebene mit der Ausblendung des Schreibens die gesamte Dimension seiner materiellen Kontiguität mit dem lebenden Menschen die Anerkennung verweigern wollte (im `Vergessen selbst, im überschreibenden Durchstreichen der wandernden Wasser des mimetischen Prozesses), so könnten wir nun umgekehrt annehmen, das Geschriebene wolle uns an uns selbst und an die hohe Schule erinnern, in die wir mit diesem Leseprozess gegangen sind. Und weil das Geschriebene die Sterne ja gar nicht be-schreiben kann, verschwindet auch das Präfix »Über-« wieder, welches über den linken unteren Stern geschrieben wurde. Sterne akzeptieren keine Tinte und keine Beschriftung. Dieses mimetische Vermögen kann nicht überschrieben werden.

Der letzte Satz von Ms 931 lautet: »Dieser Prozess würde die Überwindung des Mythos einschließen« (Ms 931). »Einschließen«, das könnte heißen, dass jene Überwindung von ihm verkapselt und unter Schloss und Riegel gehalten wird und also nicht Statt hat. Es könnte aber auch heißen, dass die Überwindung des Mythos sozusagen inbegriffen ist,

29 Friedrich Nietzsche: „Ueber Wahrheit und Lüge im aussermoralischen Sinne«, in: Kritische Studienausgabe, hg. v. Giorgio Colli/Mazzino Montinari, München - Berlin - New York (Deutscher Taschenbuch Verlag/ Walter de Gruyter) ${ }^{2} 1988$, Bd. I, S. 875-890; hier S. 883f.; (Hvh. im Original.) 
dass dieser Prozess sie also in der Tat herbeiführt. Was aber ist »dieser Prozess«? Wohl nicht die vom Text beschriebene (betriebene?) Selbst-Entfernung des die Sprache mechanisch reproduzierenden Leibes von sich selbst. Wohl schon eher dieses indexikalische Bewusstsein, das in einem erneuten Kurzschluss die beiden kleinen Sterne wieder zusammenführt, und einen neuen Funken schlägt aus der eigentlich ja sehr alten Erkenntnis, die aber doch immer wieder vergessen wird, und immer wieder neu aktualisiert werden muss, dass all jene »unendlich complicirten Begriffsdom[e]« letztlich auf »beweglichen Fundamenten und gleichsam auf fließendem Wasser« gebaut sind: auf unserer wandernden, immer neu künstlerisch schaffenden Phantasie. ${ }^{30}$

Das bloß Geschriebene kann sich nicht helfen. Ihm fehlen mindestens ein bis zwei Dimensionen, jene eben, die das Denken körperlich erlebbar machen. Die Schrift kann immer nur auf einer zweidimensionalen Fläche auseinanderlegen und somit polarisieren, was eigentlich in den Raum, in die Zeit, und in sich gehört. Beim Lesen müssen wir deshalb die polarisierten Anordnungen, welche das Geschrieben uns vorlegt, wieder rückgängig machen, und das, was da horizontal auseinandergezogen vor uns liegt - d.h. letztlich nicht nur der fünfte Abschnitt, sondern das ganze beschriftete Manuskript wieder ins Lot bringen. Wir müssen das, was im Geschriebenen sukzessive auseinanderdividiert wurde, wieder in die vertikale Schichtung zurückführen. Stellen wir uns also, zum Beispiel, einmal vor, dass die ganze Fläche jenes beschriebenen Blattes eine Konfiguration ist, und dass die horizontale Linie eine Projektion der lesbaren Fläche auf sich selbst darstellt, in dem Versuch, eine dritte Dimension, welche sie nicht hat, dennoch darzustellen. Da muss alles eben um eine Dimension reduziert werden: die Fläche wird zum Strich. Was da geschrieben stand, können wir jetzt zwar nicht mehr lesen. Dafür aber ein wenig davon, was sonst überhaupt nicht geschrieben werden konnte. Im vertikalen Schnitt fänden wir dann, oben und unten, das auseinandergelegt, was die Fläche nur ankommt, was das Blatt mit dem Geschriebenen tangiert: das mimetische Vermögen und diese Abstraktion (erster Abschnitt), die Sprache und ihre Versuche, unsere Blicke zu assimilieren (zweiter bis vierter Abschnitt), sowie der Leib, der in diesem Prozess der Lektüre sich dem Mythos entweder ergibt oder ihn überwindet (fünfter Abschnitt). Dann könnte es zu einer »Erfahrung in sich « ${ }^{31}$ kommen, einem Aufblitzen, das den

30 Ebd., S. 882.

31 Ich habe hier wiederholt auf das frühe (wahrscheinlich ca. 1918) Ms 538 "Über die Erfahrung in sich" angespielt, welches im engen Zusammenhang mit Ms 931 (vgl. Anm. 3) gelesen werden kann. Das, was ich als die hohe Schule eines indexikalisch bewussten mimetischen Prozesses diskutiert habe, ist einerseits, dass »der Lesende um keinen Preis vergessen darf, will er nicht leer ausgehen«, (GS II, S. 210). Es ist aber $\mathrm{m}$. E. auch genau das, worauf Benjamin in Ms 538 mit dem mysteriösen Wortfragment »schwinde« verweist. (Auch dieser Vergleich wird an anderer Stelle entwickelt werden müssen.) 


\section{SABINE I. GÖLZ}

verkorkten Behälter der Sprache sprengt. Dann könnte sich dieses nachträgliche Lesen als nicht weniger ursprünglich als das Schreiben erkennen. Der Kreis schließt sich, und damit ist wieder alles offen. 